\title{
Continuous monitoring of pulse period variations in Hercules X-1 using Swift/BAT
}

\author{
D. Klochkov ${ }^{1}$, R. Staubert ${ }^{1}$, K. Postnov ${ }^{2}$, N. Shakura ${ }^{2}$, and A. Santangelo ${ }^{1}$ \\ 1 Institut für Astronomie und Astrophysik, Universität Tübingen (IAAT), Sand 1, 72076 Tübingen, Germany \\ e-mail: klochkov@astro.uni-tuebingen.de \\ 2 Sternberg Astronomical Institute, Moscow University, 119992 Moscow, Russia
}

Received 30 June 2009 / Accepted 30 July 2009

ABSTRACT

\begin{abstract}
Context. Monitoring of pulse period variations in accreting binary pulsars is an important tool to study the interaction between the magnetosphere of the neutron star and the accretion disk. While the X-ray flux of the brightest X-ray pulsars have been successfully monitored over many years (e.g. with RXTE/ASM, CGRO/BATSE, Swift/BAT), the possibility to monitor their pulse timing properties continuously has so far been very limited.

Aims. In our work we show that the Swift/BAT observations can be used to monitor coherent pulsations of bright X-ray sources and use Swift archival data to study one of the most enigmatic X-ray pulsars, Hercules X-1. A quasi-continuous monitoring of the pulse period and the pulse period derivative of an X-ray pulsar, here Her X-1, is achieved over a long time ( $\gtrsim 4 \mathrm{yr}$ ). We compare our observational results with predictions of accretion theory and use them to test different aspects of the physical model of the system.

Methods. In our analysis we use the data accumulated with Swift/BAT starting from the beginning of 2005 (shortly after launch) until the present time. To search for pulsations and for their subsequent analysis we used the count rate measured by the $B A T$ detector in the entire field of view.

Results. The slope of the correlation between the locally determined spin-up rate and the X-ray luminosity is measured for Her X-1 and found to be in agreement with predictions of basic accretion torque theory. The observed behaviour of the pulse period together with the previously measured secular decrease of the system's orbital period is discussed in the framework of a model assuming ejection of matter close to the inner boundary of the accretion disk.
\end{abstract}

Key words. X-rays: binaries - stars: neutron - accretion, accretion disks

\section{Introduction}

The persistent accreting pulsar Hercules X-1 was one of the first X-ray sources discovered by the Uhuru satellite in 1972 (Tananbaum et al. 1972; Giacconi et al. 1973) and since then it remains one of the most intensively studied X-ray pulsars. The basic phenomenological picture of Her X-1 was established soon after its discovery: a close binary system consisting of an accreting magnetized neutron star with a $1.24 \mathrm{~s}$ spin period and a stellar companion HZ Her (first suggested by Liller 1972) - a main sequence star of the spectral type A/F (Crampton 1974). The mass of the optical companion is $\sim 2 M_{\odot}$ which places the system between high and low mass X-ray binaries. Other main parameters of the binary system are the following: orbital period $P_{\text {orb }} \simeq 1.7$ days, X-ray luminosity of the source $L_{\mathrm{X}} \sim 2 \times 10^{37} \mathrm{erg} \mathrm{s}^{-1}$ for a distance of $\sim 7 \mathrm{kpc}$ (Reynolds et al. 1997). The binary orbit is almost circular (Staubert et al. 2009b) and has an inclination $i \sim 85-88^{\circ}$ (Gerend \& Boynton 1976). The magnetic field strength on the surface of the neutron star is believed to be around $3 \times 10^{12} \mathrm{G}$, as estimated from the energy of the cyclotron resonant scattering feature (Trümper et al. 1978).

Like many other X-ray pulsars, Her X-1 exhibits significant variation of the pulsation period (i.e. spin rate of the neutron star). Alternation of spin-up and spin-down episodes on timescales of several months in this system is superimposed on a background of systematic spin-up (Sheffer et al. 1992; Staubert et al. 2006; Klochkov 2007). The behaviour of the pulsar's spin period on shorter time scales is not very well studied because such a study would require a continuous monitoring of Her X-1 with a sensitive X-ray detector. Only between 1991 and 2000 did the BATSE instrument onboard CGRO (Fishman et al. 1989) provide information about the source's pulse period on a regular basis. These data allowed Staubert et al. (2006) to reveal an anticorrelation between the pulse period and times of X-ray turnons, i.e. switching from an off-state with low X-ray flux to the so-called main-on state with high flux (such turn-ons regularly occur in Her X-1 with a period of $\sim 35$ days and are believed to be caused by a precessing tilted accretion disk around the neutron star, see e.g. Gerend \& Boynton 1976).

In this work we present a continuous monitoring of the Her X-1 pulse period $P$ and its local (measured at the time of the observation) time derivative $\dot{P}$ using the Swift/BAT instrument starting from 2005 (begin of scientific operation) to 2009. We compare the observed pulse period development with that measured previously with $C G R O / B A T S E$. The data of the monitoring allowed us to explore for the first time the correlation between the locally measured $\dot{P}$ and the X-ray flux of the pulsar and compare the results with predictions of accretion theory. The observed strong spin-down episodes are discussed in the framework of a model assuming ejection of matter from the inner part of the accretion disk along the open magnetic field lines.

\section{Observations}

For our analysis, we used the public archival data obtained with the Burst Alert Telescope (BAT, 15-150 keV, Barthelmy et al. 2005) onboard the Swift observatory (Gehrels et al. 2004). With 
its large field of view (1.4 sterad), the $B A T$ instrument was originally designed to provide fast triggers for gamma-ray bursts and their accurate positions in the sky $(\sim 4$ arcsec $)$. Following such a trigger, the observatory points in the direction of the burst, which can be then observed with the X-ray and UV/optical telescopes onboard the satellite. While searching for bursts, BAT points at different locations of the sky, thus performing an all-sky monitoring in hard X-rays (measurements of the X-ray flux are provided by the Swift/BAT team in the form of X-ray light curves for the several hundred bright persistent and transient sources ${ }^{1}$ ).

Most of the BAT observations are stored in the form of detector plane maps (histograms) accumulated over the 5-min exposure time, which limits the possibilities of timing analysis. In addition to the detector plane histograms, however, the stored data contain 64-ms count rates corresponding to the total flux detected by $B A T$. If a bright pulsating source with a known period falls into the field of view of the instrument, the total count rate can be used to search for coherent pulsations of that source. We have used this strategy to measure the $1.24 \mathrm{~s}$ pulsations of Her X-1 during its main-on states (when the X-ray flux of the source is high), repeating every $\sim 35$ days.

\section{Timing analysis of BAT data}

To determine the pulse period, we used a method similar to that described by Staubert et al. (2009b). The method includes two techniques for the determination of the period: epoch folding with a $\chi^{2}$ search (e.g. Leahy et al. 1983) and pulse phase connection (e.g. Deeter et al. 1981). The first one is used to establish the presence of the periodic signal from Her X-1 in the BAT data, determine the approximate period, and construct pulse profiles (by folding the data with the found period), while the second is subsequently applied to the pulse profiles to determine the precise value of the period and its time derivative.

As already mentioned, for our analysis we used the total count rates measured by BAT with a time resolution of $64 \mathrm{~ms}$. All times of the count rates were translated to the solar system barycenter and corrected for binary motion (using our newest orbital ephemeris presented in Staubert et al. 2009b). We then performed a period search using epoch folding in a narrow period interval around the expected pulse period $(\sim 1.237 \mathrm{~s})$. If a strong periodic signal was present, we determined the period and used it to construct X-ray pulse profiles for subsequent pulse phase connection. The integration time in each case was chosen to be large enough to obtain a pulse profile with sufficient statistics (normally $\sim 1 \mathrm{ks}$ ). A typical profile used in our phase-connection analysis is shown in Fig. 1.

To obtain pulse arrival times for the subsequent phase connection analysis we have fit all individual pulse profiles with a template profile constructed by superposing the individual profiles from the same main-on state. In many cases we ignored the data at the start and the end of a main-on where the profiles deviate noticeably from those obtained in the middle of the main-on. Thus, within one main-on, we restricted our analysis to the time intervals where the shape of the pulse profile does not change appreciably. Nevertheless, we cannot completely exclude possible systematic effects which might affect our results due the variation of the profile shape (see also the Discussion). If the interval between the adjacent pulse arrival times is short enough (or if the uncertainty of the assumed pulse period is sufficiently small),

\footnotetext{
${ }^{1}$ http://heasarc.gsfc.nasa.gov/docs/swift/results/ transients/
}

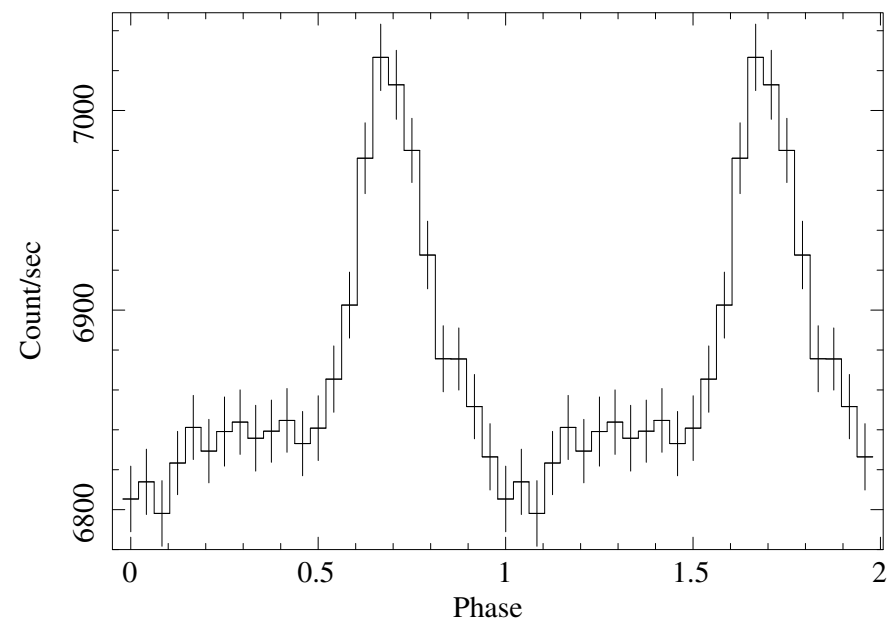

Fig. 1. An example of a Swift/BAT pulse profile used to study pulse shifts for the precise measurement of the pulse period variations in Her X-1. The profile is taken at MJD 54516.083 with $\sim 1200 \mathrm{~s}$ integration time.

one can reconstruct the number of pulsation cycles passed in between, excluding any mis-counting. In this case, the estimated pulse arrival times can be analytically modelled. For example, if the pulse period $P$ is constant, the expected arrival time of pulse number $n$ is

$t_{n}=t_{0}+n P$

where $t_{0}$ is the arrival time of the "zero"-th pulse. In the case of non-zero first and second derivatives of the pulse period, the arrival times are given by (see e.g. Staubert et al. 2009b)

$t_{n}=t_{0}+n P_{0}+\frac{1}{2} n^{2} P \dot{P}_{0}+\frac{1}{6} n^{2} P_{0}^{2} \ddot{P}_{0}+\ldots$

where $P_{0}, \dot{P}_{0}$, and $\ddot{P}_{0}$ are the pulse period and its time derivatives at the time $t_{0}$ respectively.

A convenient way to explore the variation of the pulse period, often used in the phase connection technique, is to construct a so-called $(\mathrm{O}-\mathrm{C})$ diagram showing the estimated (observed) pulse arrival time minus the calculated one assuming a constant period (i.e. using Eq. (1)). An example of such a diagram measured with BAT during one of the Her X-1 main-on states is shown in Fig. 2. A straight line in the graph would correspond to a constant period defined by the slope of the line. The solid curve indicates a parabolic fit to the data corresponding to a constant positive $\dot{P}$ according to Eq. (2) (in the shown case the best-fit $\dot{P} \simeq 1.4 \times 10^{-12} \mathrm{~s} / \mathrm{s}$ ).

\section{Pulse period variability}

Using the method described in the previous section we determined the pulse period $P$ and its time derivative $\dot{P}$ for most of the Her X-1 main-on cycles observed with $B A T$ (for other parts of the $35 \mathrm{~d}$ cycle the flux was too low for such determinations). At the time of writing this paper the data are available for the time period from March 2005 to May 2009 that covers 45 35d cycles (main-on states) of the pulsar. For several cycles the BAT observations have relatively poor statistics due to gaps in the data. For such cycles, only $P$, but no $\dot{P}$, could be determined. For the cycle with the turn-on at MJD $\sim 54339.68$, even the pulse period could not be found due to a gap in the data. However, during 


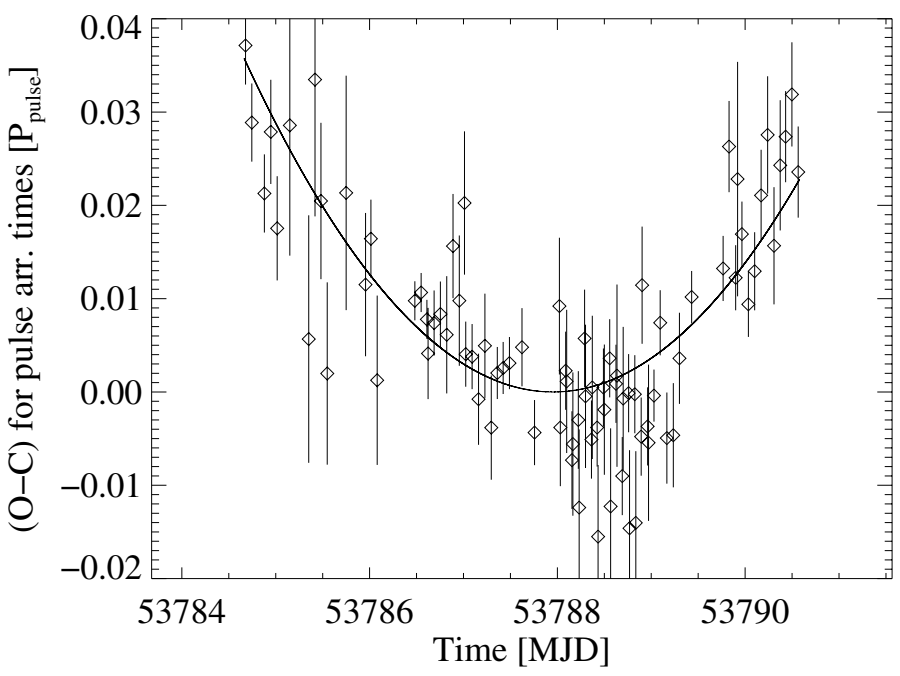

Fig. 2. Estimated (observed) minus calculated (assuming a constant period) pulse arrival times of Her X-1 in units of its pulse period during one of its main-on states observed with Swift/BAT. A parabolic fit to the data shown with the solid line corresponds to a constant positive $\dot{P} \simeq 1.4 \times 10^{-12} \mathrm{~s} / \mathrm{s}$ (according to Eq. (2)).

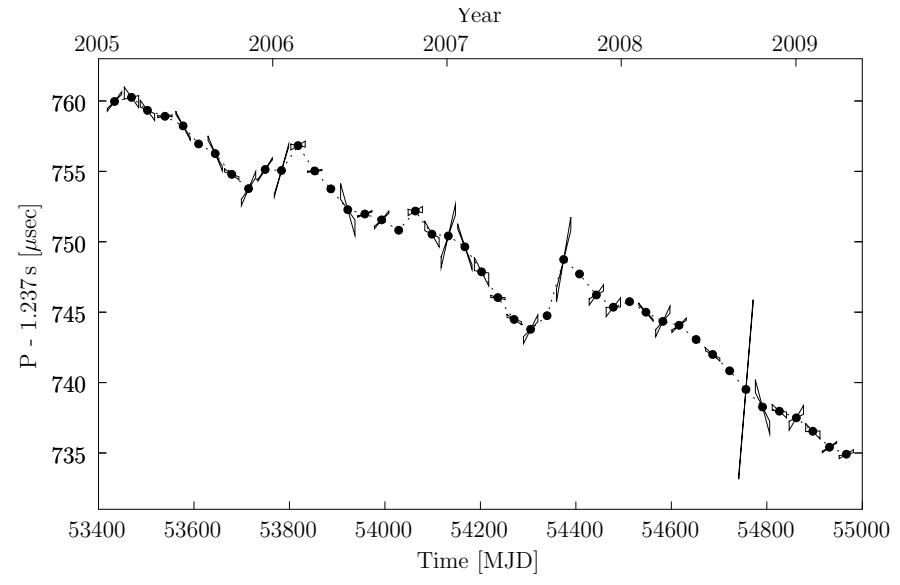

Fig. 3. Pulse period $P$ of Her X-1 measured with Swift/BAT as a function of time. The cones around each point indicate the allowed range of the slope corresponding to the measured $\dot{P}$ and its uncertainties. Measurement errors of the period itself are smaller than the symbol sizes.

this cycle Her X-1 was observed with the INTEGRAL satellite (Staubert et al. 2009b). So, we included the value of $P$ measured with INTEGRAL in our data set (since the INTEGRAL observations caught only the end of the main-on, $\dot{P}$ could not be measured).

For the main-on starting at MJD 53577.14 only the pulse period $P$ could be measured with $B A T$ (no $\dot{P}$ ). But the mainon was simultaneously observed with the INTEGRAL and RXTE observatories (Klochkov et al. 2008; Staubert et al. 2009b). The value of $P$ measured during these pointings is consistent (within $1 \sigma$ uncertainty) with the value found from the BAT data. We consider this agreement as a successful cross-check between the Swift, RXTE, and INTEGRAL observations.

The time evolution of the measured pulse period for the main-ons of Her X-1 is shown in Fig. 3. Where a corresponding value of $\dot{P}$ was measured, the $1 \sigma$-uncertainty range is indicated by the cones, the orientation of which reproduce the measured $\dot{P}$ value.
A close quantitative inspection of all measured values of $P$ and $\dot{P}$ leads to the following list of statements about their evolution with time.

- The pulse period evolution shown in Fig. 3 resembles a sawtooth where one can distinguish five spin-down and five spinup episodes (from comparing adjacent measurements). The overall mean spin-up rate is $\langle\dot{P}\rangle=-1.8203(3) \times 10^{-13} \mathrm{~s} \mathrm{~s}^{-1}$ or $-15.728(2) \mathrm{ns} \mathrm{d}^{-1}$. We note that this is slightly steeper than the general mean spin-up of $-9 \mathrm{~ns} \mathrm{~d}^{-1}$ observed from the discovery of the source to the dramatic spin-down event during the Anomalous Low of 1999/2000 (Klochkov 2007).

- The spin-down episodes (with mean spin-down rates between $0.95 \times 10^{-13}$ and $4.5 \times 10^{-13} \mathrm{~s} \mathrm{~s}^{-1}$ ), lasting from 1 to 3 $35 \mathrm{~d}$ cycles, are generally shorter than the spin-up episodes (with mean spin-up rates from $2.9 \times 10^{-13}$ to $4.4 \times 10^{-13} \mathrm{~s} \mathrm{~s}^{-1}$ ) which last from 3 to $1335 \mathrm{~d}$ cycles.

- Locally measured $\dot{P}$ values, ranging from $-11 \times 10^{-13} \mathrm{~s} \mathrm{~s}^{-1}$ to $+48 \times 10^{-13} \mathrm{~s} \mathrm{~s}^{-1}$, show stronger modulation than $\dot{P}$ values found from comparing the pulse period of adjacent $35 \mathrm{~d}$ cycles which range from $-6.0 \times 10^{-13} \mathrm{~s} \mathrm{~s}^{-1}$ to $+14 \times 10^{-13} \mathrm{~s} \mathrm{~s}^{-1}$.

- Generally, the locally measured $\dot{P}$ values and the "cycle-tocycle" $\dot{P}$ values show a similar behavior (with the former having the larger amplitude). For many points, however, the locally measured $\dot{P}$ is substantially different from the one derived from $P$ values of adjacent measurements (that is, from the slope of the pulse period development).

From the last two statements one can conclude that strong pulse period variations in Her X-1 occur on shorter time scales than the $35 \mathrm{~d}$ super-orbital period of the system.

We will discuss the pulse period variations again in Sect. 6.

\section{Correlation between spin-up rate and X-ray flux}

A positive correlation between the spin-up rate of a neutron star and its X-ray luminosity is generally expected from accretion theory (see e.g. Pringle \& Rees 1972), where an increase of the mass accretion rate $\dot{M}$ leads to an increase in the rate of change of the neutron star's angular momentum. Such a correlation has been observed in many accreting pulsars (see e.g. Bildsten et al. 1997, for a review). In Her X-1, however, the correlation was so far questionable (see however Wilson et al. 1994; Klochkov 2007). One of the difficulties is the relatively low amplitude of the $\dot{M}$ variation in the system (reflected by the maximum mainon flux, see below), of a factor $\sim 2$. Another problem is the lack of local spin-up measurements (so far $\dot{P}$ in Her X-1 was locally measured only in a few cases). For the rest of the existing data $\dot{P}$ was estimated by taking differences of the pulse periods between adjacent main-on states which gives an averaged value over two or more 35 d cycles (see e.g. Klochkov 2007, where data from CGRO/BATSE were used). The X-ray luminosity $L_{\mathrm{X}}$ which we assume to be proportional to the mass accretion rate $\dot{M}$ is usually estimated from the maximum X-ray flux during the main-on coincident with the observation (Staubert et al. 2007). Such an estimate is supposed to be very close to the local value of $L_{\mathrm{X}}$.

In this work we have used the values of $\dot{P}$ locally measured with $B A T$ to explore the correlation between the spin-up rate and the X-ray luminosity. Following Staubert et al. (2007) we have used the maximum main-on flux detected with $B A T^{2}$ as a measure of $L_{X}$. In Fig. 4 we plot the measured pulse period derivative

\footnotetext{
2 We used the Swift/BAT transient monitor results provided by the Swift/BAT team (http://heasarc.gsfc.nasa.gov/docs/swift/ results/transients/).
} 


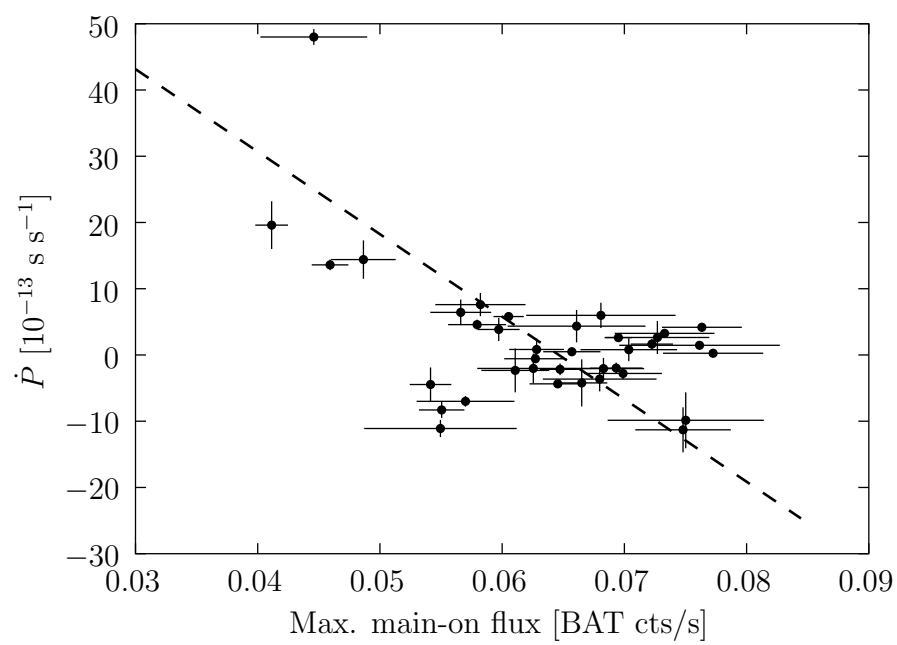

Fig. 4. The locally measured time derivative of the pulse period, $\dot{P}$, in Her X-1 versus the maximum X-ray flux during the main-on state as determined from the Swift/BAT data. A linear fit (taking uncertainties of both variables into account) is shown with the dashed line.

versus the maximum main-on flux. The dashed line indicates a linear fit to the data that takes uncertainties of both variables into account (using the orthogonal regression method, Boggs et al. 1989). The data indicate an anticorrelation as predicted by the basic accretion theory. Inspection of the linear Pearson's correlation coefficient gives a probability of $\sim 4 \times 10^{-4}$ to find the measured correlation by chance. We note, however, that such a high significance appears mainly due to the group of four points with high spin-down rate and low flux (in the upper left part of the graph in Fig. 4). The rest of the points form an uncorrelated "cloud" around $\dot{P}=0$. On the other hand, the data contain no points with large spin-down $\left(\dot{P}>10^{12} \mathrm{~s} / \mathrm{s}\right)$ and large luminosity (in the upper right part of the graph) that could destroy the correlation.

The best-fit slope of the $\dot{P}\left(L_{\mathrm{X}}\right)$ dependence assuming a linear relation is found to be $\Delta \dot{P}_{-13} / \Delta F_{\mathrm{BAT}}=-(1.2 \pm 0.2) \times 10^{3}$, where $\dot{P}_{-13}=\dot{P} /\left(10^{-13} \mathrm{~s} / \mathrm{s}\right)$ and $F_{\mathrm{BAT}}$ is the count rate measured with $B A T$. Assuming a distance of $7 \mathrm{kpc}$ (see Introduction) one can find that $0.05 B A T$ cts s$^{-1}$ approximately corresponds to $2 \times 10^{37} \mathrm{erg} \mathrm{s}^{-1}$. Thus, we can rewrite the found value of the slope as $\Delta \dot{P}_{-13} / \Delta L_{37}=-30 \pm 5$ where $L_{37}=L_{X} /\left(10^{37}\right) \mathrm{erg} \mathrm{s}^{-1}$. At a first approximation the value of the slope can be calculated using the equation:

$\frac{\mathrm{d} I \omega}{\mathrm{d} t}=\dot{M}_{x} \sqrt{G M R_{\mathrm{d}}}-\kappa_{0} \frac{\mu^{2}}{R_{\mathrm{c}}^{3}}+\kappa_{1} \mu^{2}\left(\frac{1}{R_{\mathrm{d}}^{3}}-\frac{1}{R_{\mathrm{c}}^{3}}\right)$,

where the first term stands for the spin-up from the inner disk radius $R_{\mathrm{d}}$, the second term describes spin-down due to magnetic coupling beyond the corotation radius $R_{\mathrm{c}}$, and the last term takes into account angular momentum exchange due to magnetic coupling in the region between $R_{\mathrm{c}}$ and $R_{\mathrm{d}} ; \kappa_{0}$ and $\kappa_{1}$ are numerical coefficients. The inner disk radius is about the Alfvén radius of the magnetosphere $R_{\mathrm{A}}$, which is determined in the standard way (e.g. Pringle \& Rees 1972):

$R_{\mathrm{A}}=\left(\frac{\mu^{2}}{2 \dot{M} \sqrt{2 G M}}\right)^{2 / 7}$.

At the corotational regime when $R_{\mathrm{d}} \sim R_{\mathrm{c}}$, the last term in Eq. (3) can be rewritten in the form $\kappa_{1} \mu^{2} 3\left(R_{\mathrm{c}}-R_{\mathrm{d}}\right) / R_{\mathrm{d}}^{4}$ and is much

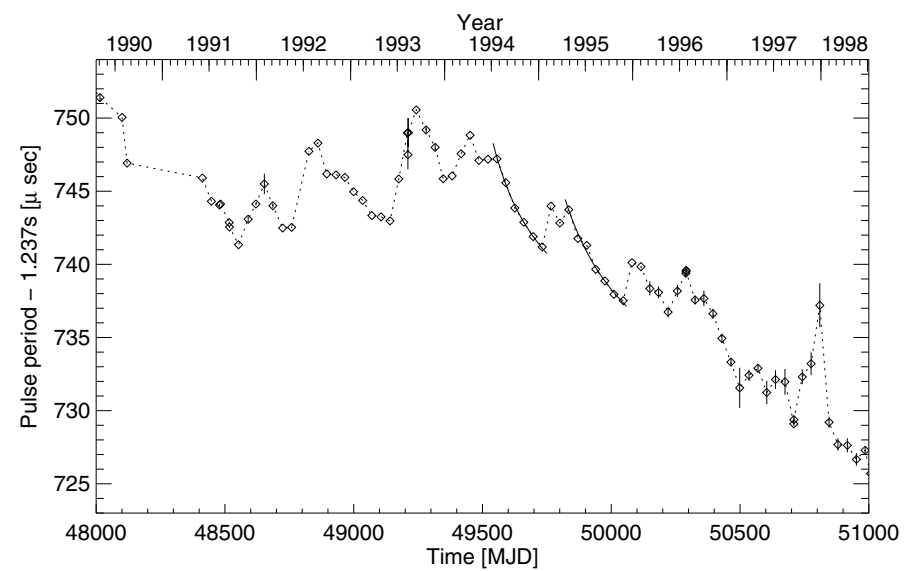

Fig. 5. Pulse period $P$ of Her X-1 measured with $C G R O / B A T S E$ as a function of time. The values are derived from the publicly available pulse search data (Wilson, priv. comm.). The solid lines indicate the exponential fit to the pulse period during the "relaxation" time after two "glitch"-like episodes.

smaller than the first two terms separately, but can be comparable to their difference at the point of equilibrium, where $R_{\mathrm{d}} \simeq R_{\mathrm{c}}$. Indeed, from Eq. (3) one can find that the spin-up and spin-down torques separately would give $\dot{P} \sim+$ or $-10^{-11} \mathrm{~s} \mathrm{~s}^{-1}$, whereas on average we observe $<\dot{P}>\sim 10^{-13} \mathrm{~s} \mathrm{~s}^{-1}$. Eq. (3) can be rewritten as

$\dot{P}=-\frac{P^{2}}{2 \pi I}\left(\dot{M} \sqrt{G M R_{\mathrm{c}}}-\kappa_{0} \frac{\mu^{2}}{R_{\mathrm{c}}^{3}}\right)$

and used to calculate the theoretical slope of the $\dot{P}\left(L_{\mathrm{X}}\right)$ dependence. Here we did not include the third term in Eq. (3), which is by $\left(R_{\mathrm{c}}-R_{\mathrm{d}}\right) / R_{\mathrm{d}}$ times smaller than the spin-up term, and can be ignored when calculating the expected slope of $\dot{P}-L_{\mathrm{X}}$ relation. Assuming a radiative efficiency of accretion of $\sim 10 \%$ $\left(L_{\mathrm{X}}=0.1 \dot{M} c^{2}\right)$ we can obtain $\partial \dot{P}_{-13} / \partial L_{37} \simeq-50$ which is close to the value of $\Delta \dot{P}_{-13} / \Delta L_{37}$ found from the fit of the $B A T$ data $(-30 \pm 5)$. A detailed discussion of the observed correlation will be given in Sects. 6.2 and 6.3.

\section{Discussion}

\subsection{Pulse period variations. Comparison with BATSE data}

The general behaviour of the pulse period in Her X-1 measured with Swift/BAT (Fig. 3) is similar to that observed previously in this source. The long-term spin-up trend is occasionally interrupted by short (a few $35 \mathrm{~d}$ cycles) spin-down episodes. In Fig. 5 we plot for comparison the historical development of the Her X-1 pulse period measured with CGRO/BATSE. We have made use of the publicly distributed pulsar data as well as lists kindly provided by Wilson (see also Staubert et al. 2006).

In both the BATSE and BAT data, the spin-down episodes are generally shorter than the spin-up episodes. One also sees that in most cases the averaged absolute value of $\dot{P}$ is larger during the spin-down intervals than during spin-ups. Such an asymmetry is difficult to explain within the basic accretion theory (i.e. using Eq. (3)) assuming that the spin period reflects stochastic variations of $\dot{M}$. In some cases spin-down episodes with subsequent spin-ups are reminiscent of glitch-like behaviour observed 
in some radio-pulsars where a rapid change of the pulse period is followed by a slower "relaxation" to the long-term trend. However, compared to radio pulsars, the "glitches" in Her X-1 appear with an opposite sign: an initial rapid increase of the period is followed by a slower decrease (as it should be if in both radio pulsars and Her X-1 the glitches are caused by a sudden decrease/increase of the moment of inertia of the star's crust). Two such glitch-like episodes are indicated in Fig. 5 by solid lines which show the fit of the "relaxation" intervals by an exponential function on top of the linear decrease of the period (see e.g. Shemar \& Lyne 1996). The characteristic relaxation times, $\tau$, for the two cases are around 100 days which is similar to the values observed in radio pulsars (Shemar \& Lyne 1996). Some of the other spin-up/spin-down transitions also resemble glitches, even though their decay is less clearly exponential. One should note, however, than in radio pulsars the glitch-like variations of the spin period are predominantly observed in cases of young neutron stars, with characteristic ages $(\tau=P / 2 \dot{P})$ less than 1 Myr. The neutron star in Her X-1 is believed to be much older.

\subsection{Correlation $\dot{P}-L_{\mathrm{X}}$}

As we have shown, the BAT data indicate a correlation between the spin-up rate $(-\dot{P})$ and the $\mathrm{X}$-ray flux $L_{\mathrm{X}}$ with the slope of the correlation consistent with the prediction of the basic accretion theory. However, even for the relatively narrow range of $L_{\mathrm{X}}$ observed in Her X-1 the correlation appears to be surprisingly loose. There are several physical and observational factors that might introduce dispersion in the observed $\dot{P}-L_{\mathrm{X}}$ relation. The observational factors include the systematics that affects our measurements of the spin-up rate and the X-ray flux. As it was already mentioned in Sect. 3, the pattern-matching technique requires a stable shape of the pulse profiles. Even though we tried to restrict our analysis to the intervals where the pulse profile does not change significantly, we cannot exclude that the variability of the profile shape contributes to the measured value of $\dot{P}$. We believe, however, that this systematic effect, if present, does not exceed the statistical errors. On the other hand, the X-ray luminosity $L_{X}$ might not be unambiguously related to the maximum main-on flux if the latter is subject to variable absorption by a hot corona even in the middle of a main-on where the angle between the plane of the accretion disk and observer's line of sight reaches a maximum.

A physical reason for the scattering in the correlation is the possible bimodal behaviour of the pulsar where it switches between the spin-up and spin-down regimes at the same level of $\mathrm{X}$-ray luminosity. Such a possibility appears e.g. in the accretion torque theory presented by Lovelace et al. (1995) where spinup/spin-down transitions occur if the corotation radius is close to the Alfvén radius (or, more precisely, to the turnover radius $r_{\text {to }}$ introduced by Lovelace et al. 1995), i.e. if the pulsar is close to the so-called corotational regime when the spin-up and spindown torques are nearly balanced.

The "uncorrelated" cloud of data points around $\dot{P}=0$ in Fig. 4 might indicate an area of the spin-up/spin-down transitions (bimodal behaviour) where different values of $\dot{P}$ are observed at the same level of $L_{X}$.

Another factor that might increase the dispersion of data points in the correlation relates to the idea of a freely precessing neutron star in Her X-1 (Trümper et al. 1978). While having a number of difficulties from the theoretical point of view (Shaham 1977; Sedrakian et al. 1999), this hypothesis, however, can explain the systematic variation of X-ray pulse profiles exhibited by the system (Staubert et al. 2009a, and references therein). Free precession results in systematic variations of the observed spin period of the neutron star (Shakura 1988; Postnov et al. 1991; Bisnovatyj-Kogan \& Kahabka 1993). With the geometrical parameters of free precession that can be assumed for Her X-1 on the basis of modelling of its pulse profiles (Postnov, priv. comm., paper in preparation), the amplitude of $\dot{P}$ variation due to free precession might be as high as $10^{-12} \mathrm{~s} / \mathrm{s}$ which is comparable to the variations that we observe with $B A T$ (Fig. 4). Since the precessional phase might be different in different observations, the described effect might introduce additional scattering in the $\dot{P}-L_{\mathrm{X}}$ correlation if the free precession indeed takes place in Her X-1.

\subsection{Evidence for the coronal mass ejection}

As mentioned in Sect. 5, in Her X-1, spin-up and spin-down torques are very well balanced, so that the averaged $\dot{P}$ is relatively close to zero. However, from the points in the upper left part of the graph in Fig. 4, one can conclude that there occur extremely large spin-down torques (at small fluxes), which are up to 5 times as strong as spin-up ones. So, the key feature to explain is why do we observe such strong spin-down episodes?

One possibility is to assume that the accretion disk carries some magnetic field which can interact via reconnection with the neutron star's magnetosphere beyond the corotation radius $R_{\mathrm{c}}$. This might imply that beyond $R_{\mathrm{c}}$ the field lines can sometimes inflate to become open (see also discussion in Lovelace et al. 1995). During such episodes, a substantial fraction of matter in the inner part of the accretion disk can escape the system in the form of a coronal wind ejection along the open field lines.

Such an ejection of matter should be reflected in a secular change of the system's orbital period which is indeed observed in Her X-1 (Deeter et al. 1991; Staubert et al. 2009b). To assess the importance of coronal mass ejections for the orbital period evolution, we invoke general considerations of the non-conservative treatment of binary orbital parameters (see e.g. Grishchuk et al. 2001). We shall assume a circular binary orbit. Let $M_{\mathrm{o}}$ be the mass of the optical star and $M_{x}$ - that of the neutron star, $q=M_{x} / M_{\mathrm{o}}$ is the binary mass ratio. The total angular momentum of the binary is mostly stored in the orbital motion of the binary components: $J=J_{\mathrm{orb}}=M_{x} M_{\mathrm{o}} /\left(M_{x}+M_{\mathrm{o}}\right) \omega_{\mathrm{orb}} a^{2}$, where $\omega_{\text {orb }}=2 \pi / P_{\text {orb }}$ is the orbital angular frequency, $P_{\text {orb }}$ is the orbital period, and $a=a_{x}+a_{\mathrm{o}}$ is the binary's semimajor axis. Let us define the non-conservativeness parameter in the standard way (e.g. Ritter \& Kolb 1992):

$\eta=-\frac{\dot{M}_{x}}{\dot{M}_{\mathrm{o}}} \leq 1, \quad \dot{M}_{\mathrm{o}}<0$.

Next we assume that the ejected mass carries away the specific angular momentum of the neutron star $j_{x}=\omega_{\text {orb }} a_{x}^{2}=$ $\omega_{\text {orb }} a^{2}\left(M_{\mathrm{o}} /\left(M_{x}+M_{\mathrm{o}}\right)\right)^{2}$. (This assumption is justified by the small inner radius of the disk $\left.R_{\mathrm{d}} \ll a_{x}\right)$. Then using the Kepler's 3rd law and the total angular momentum balance, $\dot{J}=j_{x}\left(\dot{M}_{x}+\right.$ $\dot{M}_{\text {o }}$ ), we can express the fractional change of the orbital period through $\dot{M}_{x} / M_{x}, q$ and $\eta$ :

$\frac{1}{3} \frac{\dot{P}_{\mathrm{orb}}}{P_{\mathrm{orb}}}=-\frac{\dot{M}_{x}}{M_{x}}\left[1-\frac{q}{\eta}-\left(1-\frac{1}{\eta}\right) \frac{q / 3+1}{q+1}\right]$.

For $\eta=1$ the familiar expression for the conservative mass exchange is obtained. It is convenient to normalize the mass accretion rate onto the neutron star $\dot{M}_{x}$ to the value that can be derived 
from the observed fractional change of the orbital period in the conservative case:

$$
\frac{\left(\dot{M}_{x}\right)_{\text {cons }}}{M_{x}}=\frac{1}{3} \frac{\dot{P}_{\text {orb }}}{P_{\text {orb }}} \frac{1}{q-1},
$$

so that $\dot{m} \equiv \dot{M}_{x} /\left(\dot{M}_{x}\right)_{\text {cons. }}$ In Her X-1 $q \simeq 0.63$ and one finds $\left(\dot{M}_{x}\right)_{\text {cons }} \simeq 8 \times 10^{17} \mathrm{~g} \mathrm{~s}^{-1}$ for the measured $\dot{P}_{\text {orb }}=$ $-4.85 \times 10^{-11} \mathrm{~s} \mathrm{~s}^{-1}$ (Staubert et al. 2009b). Then we can eliminate $\dot{P}_{\text {orb }} / P_{\text {orb }}$ from the left hand side of Eq. (7) to obtain the equation for $\eta$ at a given $\dot{m}$ :

$\eta=\frac{q^{2}+\frac{2}{3} q-1}{\frac{q^{2}-1}{\dot{m}}+\frac{2}{3} q}$.

From here we see that $\dot{m}<1$ leads to $\eta<1$, i.e. if one wants to decrease the mass accretion rate onto the neutron star to get a smaller X-ray luminosity (as is the case of Her X-1, where the mean observed $L_{x} \sim 2 \times 10^{37} \mathrm{erg} \mathrm{s}^{-1}$ is 4 times smaller than the one following from the conservative mass exchange analysis, Staubert et al. 2009b), a non-conservative mass exchange is required. Specifically, if we want to bring into accordance the observed $\dot{P}_{\text {orb }}$ and $L_{\mathrm{X}}$ in Her X-1, we would need $\dot{m} \simeq 1 / 4$ and (from Eq. (9)) $\eta \sim 0.1$, a fairly high non-conservative mass exchange.

The binary mass ratio $q$ in Her $\mathrm{X}-1$, however, may be uncertain. Indeed, recent analysis of non-LTE effects in the formation of the $H_{\gamma}$ absorption line allows two solutions: $q=0.45$ and $q=0.72$ (Abubekerov et al. 2008). Equation (8) implies that the critical $\left(\dot{M}_{x}\right)_{\text {cons }}$ decreases for smaller $q$ : for $q=0.45$ we obtain $\left(\dot{M}_{x}\right)_{\text {cons }} \simeq 5.4 \times 10^{17} \mathrm{~g} \mathrm{~s}^{-1}$. For this mass ratio the appropriate value of the dimensionless parameter $\dot{m}$ is $1 / 2$, for which from Eq. (9) we find $\eta \simeq 0.38$. This value implies that on average about half of the matter transferred through the disk should escape from the system to provide the observed decrease of the system's orbital period. Note, however, that such a small mass ratio suggests an unusually small mass of the neutron star of about $0.85 M_{\odot}$.

It is very likely that an accretion disk corona is present in Her X-1 (see the recent analysis of Chandra X-ray observations by Ji et al. 2009). This suggests that there might be a permanent coronal accretion disk wind which carries away some angular momentum from the system. Episodic ejection of matter in Her $\mathrm{X}-1$ has sporadically been invoked in the literature to explain some properties of the system. Crosa \& Boynton (1980) found that the average mass transfer rate to the outer rim of the accretion disk is somewhat larger than that required to maintain the observed X-ray luminosity. In the model of Schandl \& Meyer (1994), the disk wind results from irradiation of the disk by the central source. Vrtilek et al. (2001) and Boroson et al. (2001) found signatures of outflowing gas in the UV spectrum of the system. In the framework of our model, the mass ejection from the system through the open magnetic field lines occurs most efficiently during strong spin-down episodes which are associated with low X-ray luminosity. Indeed, as we see in Fig. 4, the observed X-ray flux is decreased by a factor of two during strong spin-down. From Eq. (9) it is easy to find that at a given $q$ a fractional decrease in $\dot{m}$ leads to a comparable fractional decrease in $\eta$, i.e. accretion indeed becomes more non-conservative during strong mass ejection episodes. During such episodes, the neutron star spin-down power $I \omega \dot{\omega}$ is spent to expel accreting matter from the inner disk radius $R_{\mathrm{d}} \sim R_{\mathrm{c}}$ :

$I \omega \dot{\omega} \sim \dot{M}_{\mathrm{ej}} \frac{G M}{R_{\mathrm{c}}}$.
This equation is satisfied for the observed parameters of Her X-1: the ejected mass rate during strong spin-downs $\dot{M}_{\mathrm{ej}} \sim$ $0.5 \dot{M}_{x} \simeq 10^{17} \mathrm{~g} \mathrm{~s}^{-1}, \dot{P} \simeq 10^{-12} \mathrm{~s} \mathrm{~s}^{-1}$, and $R_{\mathrm{c}} \simeq 1.3 \times 10^{8} \mathrm{~cm}$.

\section{Summary}

We have used the publicly available Swift/BAT data to study the long-term behaviour of the pulsar's spin period in Her X-1. The measured pulse period variations were compared with those observed previously with CGRO/BATSE.

For the first time, the pulse period derivative was measured for a long series of observed main-on states of the source. This allowed us for the first time to test the correlation between the $\mathrm{X}$-ray luminosity and the locally measured spin-up rate of the neutron star in Her X-1. We argue that the data indeed show such a correlation with the slope consistent with the prediction of the basic accretion theory for the parameters of Her X-1. The relatively large scattering of the data points in the vicinity of $\dot{P} \sim 0$ can be caused by the bimodal behaviour of the accretion flow configuration at the magnetospheric boundary which results in switching of the pulsar between spin-up and spin-down branches when it stays close to the corotational regime. In addition, free precession of the neutron star, if it takes place in the system, can significantly affect the measured values of $\dot{P}$ and, therefore, contribute to the scattering.

We argue that together with the long-term decrease of the orbital period in Her X-1 the measured pulse period behaviour requires the presence of mass ejection from the inner parts of the accretion disk along the open magnetic field lines. The mass ejection episodes probably take place during strong spin-down episodes which are associated with the low X-ray luminosity.

The described technique allows one to use the BAT instrument as a long-term monitor of spin periods in other bright accreting pulsars (see also Camero-Arranz et al. 2009).

Acknowledgements. The work was supported by the DLR grant BA5027, RFBR grant 09-02-00032, and DFG grants Sta 173/31 and RUS 113/717/0-1

We thank ISSI (Bern, Switzerland) for its hospitality during the team meetings of our collaboration when we discussed the presented results.

D.K. thanks Valery Suleimanov (IAAT, Tübingen) for useful discussions.

\section{References}

Abubekerov, M. K., Antokhina, E. A., Cherepashchuk, A. M., et al. 2008, Astron. Rep., 52, 379

Barthelmy, S. D., Barbier, L. M., Cummings, J. R., et al. 2005, Space Sci. Rev., 120, 143

Bildsten, L., Chakrabarty, D., Chiu, J., et al. 1997, ApJS, 113, 367

Bisnovatyj-Kogan, G. S., \& Kahabka, P. 1993, A\&A, 267, L43

Boggs, P. T., Donaldson, J. R., Byrd, R. H., et al. 1989, ACM Trans. Math. Softw., 15, 348

Boroson, B., Kallman, T., \& Vrtilek, S. D. 2001, ApJ, 562, 925

Camero-Arranz, A., Finger, M. H., Ikhsanov, N. R., Wilson-Hodge, C. A., \& Beklen, E. 2009, ArXiv e-prints

Crampton, D. 1974, ApJ, 187, 345

Crosa, L., \& Boynton, P. E. 1980, ApJ, 235, 999

Deeter, J. E., Boynton, P. E., \& Pravdo, S. H. 1981, ApJ, 247, 1003

Deeter, J. E., Boynton, P. E., Miyamoto, S., et al. 1991, ApJ, 383, 324

Fishman, G. J., Meegan, C. A., Wilson, R. B., et al. 1989, in BAAS, 21, 860

Gehrels, N., Chincarini, G., Giommi, P., et al. 2004, ApJ, 611, 1005

Gerend, D., \& Boynton, P. E. 1976, ApJ, 209, 562

Giacconi, R., Gursky, H., Kellogg, E., et al. 1973, ApJ, 184, 227

Grishchuk, L. P., Lipunov, V. M., Postnov, K. A., Prokhorov, M. E., \& Sathyaprakash, B. S. 2001, Physics Uspekhi, 44, 1 
Ji, L., Schulz, N., Nowak, M., Marshall, H. L., \& Kallman, T. 2009, ArXiv e-prints

Klochkov, D. 2007, Ph.D. Thesis, University of Tübingen, Germany, http:// w210.ub.uni-tuebingen. de/volltexte/2007/3181/pdf/disser. $\operatorname{pdf}$

Klochkov, D., Staubert, R., Postnov, K., et al. 2008, A\&A, 482, 907

Leahy, D. A., Elsner, R. F., \& Weisskopf, M. C. 1983, ApJ, 272, 256

Liller, W. 1972, IAU Circ., 2415

Lovelace, R. V. E., Romanova, M. M., \& Bisnovatyi-Kogan, G. S. 1995, MNRAS, 275, 244

Postnov, K. A., Prokhorov, M. E., \& Shakura, N. I. 1991, in High-Energy Astrophysics. American and Soviet Perspectives, ed. W. H. G. Lewin, G. W. Clark, R. A. Sunyaev, K. K. Trivers, \& D. M. Abramson, 307

Pringle, J. E., \& Rees, M. J. 1972, A\&A, 21, 1

Reynolds, A. P., Quaintrell, H., Still, M. D., et al. 1997, MNRAS, 288, 43

Ritter, H., \& Kolb, U. 1992, A\&A, 259, 159

Schandl, S., \& Meyer, F. 1994, A\&A, 289, 149

Sedrakian, A., Wasserman, I., \& Cordes, J. M. 1999, ApJ, 524, 341

Shaham, J. 1977, ApJ, 214, 251
Shakura, N. I. 1988, in Proc. "Physics of Neutron Stars", Leningrad, 2, english translation: Physics of Neutron Stars, ed. A. M. Kaminker, Nova, Sci. Publ., Inc., 55, 1995

Sheffer, E. K., Kopaeva, I. F., Averintsev, M. B., et al. 1992, Sov. Astron., 36, 41 Shemar, S. L., \& Lyne, A. G. 1996, MNRAS, 282, 677

Staubert, R., Schandl, S., Klochkov, D., et al. 2006, in The Transient Milky Way: A Perspective for MIRAX, ed. F. D'Amico, J. Braga, \& R. E. Rothschild, AIP Conf. Ser., 840, 65

Staubert, R., Shakura, N. I., Postnov, K., et al. 2007, A\&A, 465, L25

Staubert, R., Klochkov, D., Postnov, K., et al. 2009a, A\&A, 494, 1025

Staubert, R., Klochkov, D., \& Wilms, J. 2009b, A\&A [arXiv:0904.2307], Accepted

Tananbaum, H., Gursky, H., Kellogg, E. M., et al. 1972, ApJ, 174, L143

Trümper, J., Pietsch, W., Reppin, C., et al. 1978, ApJ, 219, L105

Vrtilek, S. D., Quaintrell, H., Boroson, B., et al. 2001, ApJ, 549, 522

Wilson, R. B., Finger, M. H., Pendleton, G. N., Briggs, M., \& Bildsten, L. 1994, in The Evolution of X-ray Binariese, ed. S. Holt, \& C. S. Day, AIP Conf. Ser., 308475 